\title{
Prevalencia de Dolor Miofascial en Músculos de la Masticación y Cervicales en un Centro Especializado en Trastornos Temporomandibulares y Dolor Orofacial
}

\author{
Prevalence of Myofascial Pain in Masticatory and Neck Muscles in a \\ Specialized Center of Temporomandibular Disorders and Orofacial Pain
}

Iturriaga, V."; Bornhardt, T.; Hermosilla, L.* \& Avila, M.*

ITURRIAGA, V.; BORNHARDT, T.; HERMOSILLA, L. \& AVILA, M. Prevalencia de dolor miofascial en músculos de la masticación y cervicales en un centro especializado en trastornos temporomandibulares y dolor orofacial. Int. J. Odontostomat., 8(3):413-417, 2014.

RESUMEN: El dolor miofascial es una patología muscular regional no inflamatoria que en el territorio craneocervical se encuentra clasificada dentro de los Trastornos Temporomandibulares. Afecta al 95\% de las personas con trastornos de dolor crónico y es un hallazgo común en pacientes en tratamiento en centros de dolor. Se revisaron 121 fichas de pacientes atendidos en el policlínico de Trastornos Temporomandibulares y Dolor Orofacial de la Universidad de La Frontera, TemucoChile, identificando la prevalencia de dolor miofascial y su asociación con la edad y sexo de los pacientes. El 80,99\% de los pacientes presentó dolor miofascial, el músculo masetero fue el más afectado, seguido por los músculos temporal y trapecio superior, siendo frecuente la afectación bilateral y simultánea de diferentes músculos. La presencia de dolor miofascial no se asocia a la edad de los pacientes pero si al sexo de estos, donde las mujeres presentan más frecuentemente esta patología. Es importante realizar un correcto diagnóstico de dolor miofascial en pacientes que acuden por dolor en este territorio y más aún es trascendental tomar conciencia de su elevada prevalencia.

PALABRAS CLAVE: síndrome dolor miofascial, trastornos temporomandibulares, disfunción temporomandibular, puntos gatillo miofasciales, prevalencia.

\section{INTRODUCCIÓN}

El Dolor Miofascial por puntos gatillo (DMF) descrito por Travell \& Simons (Simons, 2002) corresponde a una patología muscular regional no inflamatoria que se puede presentar en cualquier músculo estriado del cuerpo (Aragón et al., 2005; Vázquez-Delgado et al., 2009). Su característica principal es la presencia de una zona hipersensible denominada punto gatillo (Aragón et al.; Okeson, 2008), el cual se encuentra presente en una banda tensa palpable de tejido muscular (Martinez \& Pecos, 2005; Okeson) y posee la capacidad de referir dolor a zonas distantes (Simons et al., 2002). Esta última cualidad hace que su diagnóstico se dificulte y puede ser motivo de tratamientos fallidos.

EI DMF es una patología frecuente en la población general (Carames et al., 2009; Simons et al.), llegándose a pensar que estaría presente en la vida de todos en algún momento (Simons et al.; Picavet et al., 2000). En el territorio orofacial y cervical el dolor miofascial representa la causa más común de dolor muscular siendo diagnóstico diferencial en el $60 \%$ de los casos de dolor de la articulación temporomandibular (Aragón et al.), presentando una incidencia en los músculos de la masticación del 25\% (Wright, 2000). Afecta a un $95 \%$ de las personas con un trastorno de dolor crónico y es un hallazgo común en centros especializados de dolor (Shah et al., 2008).

En la población general la edad más frecuente de presentación varía entre los 27,5 años y los 50 años (Vázquez-Delgado et al.) y la incidencia alcanza al 54\% en mujeres y el $45 \%$ en hombres (Wright). Esta última diferencia es controversial ya que en algunos estudios la diferencia es estadísticamente significativa y 
en otros no (Okeson; Simons et al.). Al ser una patología común y con gran impacto en la calidad de vida es importante determinar su frecuencia en clínicas especializadas de manejo de dolor, tomando en cuenta que el DMF tiene un carácter crónico y su manejo puede ser complejo (Valmaseda \& Gay Escoda, 2002).

\section{MATERIAL Y MÉTODO}

El estudio fue aprobado por el Comité Ético Científico de la Universidad de La Frontera (Resolución exenta $n^{\circ}$ 4204). Se realizó un estudio de corte transversal donde se revisaron las fichas de los pacientes atendidos en el policlínico de Trastornos Temporomandibulares y Dolor Orofacial de la Universidad de La Frontera, Temuco- Chile, desde junio de 2010 hasta diciembre de 2012, identificándose las variables edad, sexo y DMF.

Se incluyeron en el estudio todos los pacientes cuyas fichas tuvieran consignadas las variables en estudio y se excluyeron los pacientes que presentaran un diagnóstico previo de fibromialgia.

Dolor Miofascial. La evaluación de dolor miofascial fue realizada en todos los casos por un especialista en Trastornos Temporomandibulares y Dolor Orofacial perteneciente al Policlínico de Trastornos Temporomandibulares y Dolor Orofacial de la Universidad de La Frontera, Temuco - Chile. El especialista fue entrenado para realizar una presión de medio kilo $(0,5 \mathrm{~K})$ en cada músculo, en posición de reposo. El diagnóstico de DMF se obtuvo a través de los criterios diagnósticos escenciales propuestos por Simons et al.

Se consideró un diagnóstico positivo de DMF al cumplir con al menos 3 de los cuatro criterios diagnósticos escenciales, quedando consignado en la ficha del paciente. Los músculos evaluados fueron masetero, temporal, trapecio en su porción superior, esplenio de la cabeza y esternocleidomastoideo. Todos los músculos fueron examinados de forma bilateral.

Análisis estadístico. El análisis estadístico se realizó utilizando el programa de software SPSS/PC + versión 20.0, SPSS, Chicago, IL. La estadística descriptiva se calculó para las diferentes mediciones, Media \pm desviación estándar. La normalidad de la distribución se comprobó mediante la prueba de Kolgomorov-Smirnov. Para el análisis de las variables cualitativas se utilizó la prueba Chi-Cuadrado $\left(X^{2}\right)$ para establecer si existe asociación entre dichas variables. Para el análisis de homogeneidad de varianzas se utilizó la prueba de Levene y para la diferencia de medias poblacionales se utilizó la prueba de $U$ de MannWhitney. El valor $p<0,05$ fue elegido como umbral de significación estadística.

\section{RESULTADOS}

Se revisaron 121 fichas que cumplían los criterios de inclusión y exclusión donde el promedio de edad de los pacientes fue 26,98 (DE 16,51) años, siendo los 15 años la moda. El $76,86 \%$ de los pacientes fue de sexo femenino $(n=93)$ y el $23,14 \%$ de sexo masculino $(n=28)$, no existiendo diferencias significativas entre las medias poblacionales de las edades según el sexo de los pacientes $(p=0,187)$.

En relación a la presencia de DMF, del total de pacientes el $80,99 \%(n=98)$ de los pacientes presento DMF en alguno de los músculos estudiados. La edad no fue un factor asociado $(p=0,396)$, a diferencia del sexo, donde las mujeres presentan significativamente más DMF $(p=0,043)$. En la Tabla I se detalla la presencia de DMF según el sexo de los pacientes.

En el grupo muscular masticatorio el músculo masetero izquierdo presentó mayor frecuencia de

Tabla I. Frecuencia de dolor miofascial según sexo de los pacientes.

\begin{tabular}{lcccc}
\hline \multirow{2}{*}{ Sexo } & \multicolumn{3}{c}{ DMF } \\
\cline { 2 - 5 } & & Presente & Ausente & Total \\
\hline Mujer & $\mathrm{n}$ & 79 & 14 & 93 \\
& $\%$ & 80,61 & 60,87 & 76,86 \\
Hombre & $\mathrm{n}$ & 19 & 9 & 28 \\
& $\%$ & 19,39 & 39,13 & 23,14 \\
Total & $\mathrm{n}$ & 98 & 23 & 121 \\
& $\%$ & 100,00 & 100,00 & 100,00 \\
\hline \multicolumn{2}{l}{ DMF= Dolor miofascial; $n=$ Número de pacientes; $\%=$ porcentaje del total. }
\end{tabular}


Tabla II. Prevalencia de dolor miofascial según lado afectado en los músculos masetero, temporal, trapecio superior, esplenio de la cabeza y esternocleidomastoideo.

\begin{tabular}{lcccc}
\hline \multirow{2}{*}{ Músculo } & \multicolumn{2}{c}{ Derecho } & \multicolumn{2}{c}{ Izquierdo } \\
\cline { 2 - 5 } & $\mathbf{n}$ & $\mathbf{\%}$ & $\mathbf{n}$ & $\mathbf{\%}$ \\
\hline Masetero & 83 & 68,59 & 87 & 71,90 \\
Temporal & 79 & 65,29 & 76 & 62,81 \\
Trapecio superior & 71 & 58,68 & 61 & 50,41 \\
Esplenio de la cabeza & 43 & 35,54 & 36 & 29,75 \\
Esternocleidomastoideo & 63 & 52,07 & 59 & 48,76 \\
\hline
\end{tabular}

$\mathrm{n}=$ número de pacientes afectados; \%= porcentaje de pacientes afectados del total de pacientes.

DMF, estando afectado en un $71,90 \%$ de los pacientes $(n=87)$, representando el músculo más afectado entre todos los estudiados. Entre los músculos cervicales el que presento mayor frecuencia de DMF fue el músculo trapecio superior derecho con un $58,68 \%(n=71)$. En la Tabla II se muestran las frecuencias por cada músculo estudiado.

El músculo masetero fue el de mayor frecuencia de afectación bilateral con un $64,46 \%(n=78)$ (Tabla III).

Tabla III. Prevalencia de dolor miofascial de forma bilateral en los músculos masetero, temporal, trapecio superior, esplenio de la cabeza y esternocleidomastoideo.

\begin{tabular}{lcc}
\hline Músculo & $\mathbf{n}$ & $\mathbf{\%}$ \\
\hline Masetero & 78 & 64,46 \\
Temporal & 70 & 57,85 \\
Trapecio superior & 61 & 50,41 \\
Esplenio de la cabeza & 35 & 28,92 \\
Esternocleidomastoideo & 53 & 43,80 \\
\hline n= número de pacientes afectados; \% & porcentaje de pa- \\
cientes afectados del total de pacientes.
\end{tabular}

\section{DISCUSIÓN Y CONCLUSIONES}

La prevalencia de DMF es alta en los pacientes que consultan por dolor orofacial y trastornos temporomandibulres, siendo del $80,99 \%$. Se ha descrito una prevalencia del $44 \%$ en la población americana (Wheeler, 2004), siendo la edad más frecuente de presentación entre los 27,5 y 50 años. Esto último no coincide con los resultados encontrados, ya que la edad más frecuente de presentación es a los 15 años, pero apoya la idea de que el DMF no esta- ría asociado a la edad de presentación de la patología, pudiendo debutar en cualquier momento. Por otro lado la presencia de DMF mayoritariamente en mujeres es controversial. Al parecer existirían factores asociados que contribuyen a esta asociación, como lo son la calidad de sueño, donde mujeres con afecciones músculo-esqueléticas tienen un sueño de menor duración y con más despertares en comparación a los hombres con la misma patología (Lavigne et al., 2011), lo que podría causar que el dolor se perpetúe y aumente (Martín-Herrero et al., 2012).

Al observar la cantidad de pacientes afectados por DMF se evidencia una alta prevalencia en todos los músculos estudiados, siendo la menor de un $29,75 \%$ (m. esternocleidomastoideo izquierdo) y la mayor $71,90 \%$ (m. masetero izquierdo), además de la gran afectación de varios músculos simultáneamente y de forma bilateral. Se ha asociado la presencia de DMF a otras condiciones dolorosas como la cefalea tipo tensional (Fernández-de-lasPeñas et al., 2007a; Alonso-Blanco et al. 2011), encontrándose una mayor asociación con el músculo trapecio superior (Fernández-de-las-Peñas et al., 2006), he incluso se ha llegado a cuestionar la fisiopatología de esta cefalea primaria (Fernándezde-las-Peñas, 2007b). De igual forma se han encontrado altas prevalencias de DMF en pacientes con dolor cervical mecánico, lesiones en latigazo, cefalea cervicogénica (Fernández-del-las-Peñas et al., 2007c; De-la-Llave-Rincon et al., 2012) y fibromialgia (Alonso-Blanco et al., 2012), lo que hace aún más importante el estudio del DMF pensando en la gran cantidad de ausencias laborales e incapacidad que se produce en los pacientes. Esto muestra la importancia del correcto diagnóstico en pacientes que acuden por dolor en este territorio y lo trascendental de tomar conciencia de su elevada prevalencia. 
ITURRIAGA, V.; BORNHARDT, T.; HERMOSILLA, L. \& AVILA, M. Prevalencia de dolor miofascial en músculos de la masticación y cervicales en un centro especializado en trastornos temporomandibulares y dolor orofacial. Int. J. Odontostomat., 8(3):413-417, 2014

ITURRIAGA, V.; BORNHARDT, T.; HERMOSILLA, L. \& AVILA, M. Prevalence of myofascial pain in masticatory and neck muscles in a specialized center of temporomandibular disorders and orofacial pain. Int. J. Odontostomat., 8(3):413-416, 2014.

ABSTRACT: Myofascial pain is a noninflammatory regional muscle pathology, in craniocervical territory it is classified as temporomandibular disorder. Myofascial pain affects $95 \%$ of people with chronic pain disorders and is a common finding in pain centers. One hundred twenty-one charts of patients seen at the clinic of Temporomandibular Disorders and Orofacial Pain at the Universidad de La Frontera Temuco-Chile, were reviewed, identifying the prevalence of myofascial pain and its association with age and sex of the patients. In $80.99 \%$ of patients myofascial pain was present, the masseter muscle was the most affected, followed by the temporal and upper trapezius muscles, with frequent bilateral and simultaneous involvement of different muscles. The presence of myofascial pain is not associated with patient age but it is associated with the sex of these, where women have this condition more often. It is important to make a correct diagnosis of myofascial pain in patients presenting with pain in this area and it is even more crucial to be aware of its high prevalence.

KEY WORDS: myofascial pain syndromes, temporomandibular joint dysfunction syndrome, myofascial trigger points, temporomandibular joint disorders, frequency.

\section{REFERENCIAS BIBLIOGRÁFICAS}

Alonso-Blanco, C.; Fernández-de-las-Peñas, C.; de-la-Llave-Rincón, A. I.; Zarco-Moreno, P.; Galán-del-Río, F. \& Svensson, P. Characteristics of referred muscle pain to the head from active trigger points in women with myofascial temporomandibular pain and fibromyalgia syndrome. J. Headache Pain, 13(8):625-37, 2012.

Alonso-Blanco, C.; Fernández-de-las-Peñas, C.; Fernández-Mayoralas, D. M.; de-la-Llave-Rincón, A. I.; Pareja, J. A. \& Svensson, P. Prevalence and anatomical localization of muscle referred pain from active trigger points in head and neck musculature in adults and children with chronic tension-type headache. Pain Med., 12(10):1453-63, 2011.

Aragón, M. C.; Aragón, F. \& Torres L. M. Trastornos de la articulación témporo-mandibular. Rev. Soc. Esp. Dolor, 12(7):429-35, 2005.

Carames, J.; Carvalhão, F. \& Real Dias, M. C. Dor miofascial por pontos gatilho, uma doença multidisciplinar. Acta Reumatol. Port., 34(1):38-43, 2009.

De-la-Llave-Rincón, A. I.; Alonso-Blanco, C.; Gil-Crujera, A.; Ambite-Quesada, S.; Svensson, P. \& Fernándezde-las-Peñas, C. Myofascial trigger points in the masticatory muscles in patients with and without chronic mechanical neck pain. J. Manipulative Physiol. Ther., 35(9):678-84, 2012.

Fernández-de-las-Peñas, C.; Alonso-Blanco, C.; Cuadrado, M. L.; Gerwin, R. D. \& Pareja, J. A. Myofascial trigger points and their relationship to headache clinical parameters in chronic tension-type headache. Headache, 46(8):1264-72, 2006.
Fernández-de-las-Peñas, C.; Ge, H. Y.; Arendt-Nielsen, L.; Cuadrado, M. L. \& Pareja, J. A. The local and referred pain from myofascial trigger points in the temporalis muscle contributes to pain profile in chronic tension-type headache. Clin. J. Pain, 23(9):786-92, 2007a.

Fernández-de-las-Peñas, C.; Cuadrado, M. L.; ArendtNielsen, L.; Simons, D. G. \& Pareja, J. A. Myofascial trigger points and sensitization: an updated pain model for tension-type headache. Cephalalgia, 27(5):383-93, $2007 b$.

Fernández-de-las-Peñas, C.; Simons, D. G.; Cuadrado, M. L. \& Pareja, J. A. The role of myofascial trigger points in musculoskeletal pain syndromes of the head and neck. Curr. Pain Headache Rep., 11(5):365-72, 2007c.

Lavigne, G. J.; Okura, K.; Abe, S.; Colombo, R.; Huynh, N.; Montplaisir, J. Y.; Marchand, S. \& Lanfranchi, P. A. Gender specificity of the slow wave sleep lost in chronic widespread musculoskeletal pain. Sleep Med., 12(2):179-85, 2011.

Martín-Herrero, C.; Rodrigues de Souza, D. P.; Alburquerque-Sendín, F.; Ortega-Santiago, R. \& Fernández-de-Las-Peñas, C. Puntos gatillo miofasciales, dolor, discapacidad y calidad del sueño en pacientes con cefalea de tensión crónica: estudio piloto. Rev. Neurol., 55(4):193-9, 2012.

Martínez, C. J. M. \& Pecos, M. D. Criterios diagnósticos y características clínicas de los puntos gatillo miofasciales. Fisioter., 27(2):65-8, 2005. 
ITURRIAGA, V.; BORNHARDT, T.; HERMOSILLA, L. \& AVILA, M. Prevalencia de dolor miofascial en músculos de la masticación y cervicales en un centro especializado en trastornos temporomandibulares y dolor orofacial. Int. J. Odontostomat., 8(3):413-417, 2014.

Okeson, J. P. Tratamiento de oclusión y afecciones temporomandibulares. $6^{a}$ ed. Madrid, Elsevier, 2008.

Picavet, H. S. J.; Van Gils, H. W. V. \& Schouten, J. S. A. G. Musculoskeletal complaints in the Dutch population. The Netherlands, RIVM (National Institute of Public Health and the Environment, 2000.

Shah, J. P.; Danoff, J. V.; Desai, M. J.; Parikh, S.; Nakamura, L. Y.; Phillips, T. M. \& Gerber, L. H. Biochemicals associated with pain and inflammation are elevated in sites near to and remote from active myofascial trigger points. Arch. Phys. Med. Rehabil., 89(1):16-23, 2008.

Simons, D. G.; Travell, J. G. \& Simons, L. S. Dolor y disfunción miofascial. El manual de los puntos gatillo. Mitad superior del cuerpo. 2. ${ }^{a}$ ed. Madrid, Médica Panamericana, 2002.

Valmaseda, E. \& Gay Escoda, C. Diagnóstico y tratamiento de la patología de la articulación temporomandibular. ORL-DIPS, 29(2):55-70, 2002.

Vázquez-Delgado, E.; Cascos-Romero, J. \& Gay-Escoda, C. Myofascial pain syndrome associated with trigger points: a literature review. (I): Epidemiology, clinical treatment and etiopathogeny. Med. Oral Patol. Oral Cir. Bucal, 14(10):e494-8, 2009.

Wheeler, A. H. Myofascial pain disorders: theory to therapy. Drugs, 64(1):45-62, 2004.

Wright, E. F. Referred craniofacial pain patterns in patients with temporomandibular disorder. J. Am. Dent. Assoc., 131(9):1307-15, 2000.
Dirección para Correspondencia:

Veronica Iturriaga, DDS, MSc

Departamento de Odontología Integral del Adulto

Facultad de Odontología

Universidad de La Frontera

Claro Solar 115

Temuco

CHILE

Email: veronica.iturriaga@ufrontera.cl

Recibido : 13-03-2014

Aceptado: 12-11-2014 